\title{
Small Bowel Disease
}

\author{
Andrea Laghi and Amy K. Hara
}

\section{Learning Objectives}

- To understand the role of different imaging modalities in small bowel diseases

- To learn the most common CT findings in the emergency setting, including small bowel obstruction and ischemia

- To learn the relevant CT and MR findings in the diagnosis of Crohn's disease, according to different disease phenotypes

- To be aware of small bowel injury induced by nonsteroidal anti-inflammatory drugs (NSAID)

- To learn imaging findings in celiac disease

- To learn how to perform a differential diagnosis among different small bowel tumors

\subsection{Techniques (US, CTE, MRE)}

Ultrasonography (US), magnetic resonance (MR), and computed tomography (CT) have a complementary role in the evaluation of small bowel disorders [1].

US is a powerful tool for bowel assessment in patients with known or suspected gut-related disease, especially in combination with oral (SICUS) [2] and intravenous (CEUS) contrast agents [3]. It is often the first imaging modality used in patients with undiagnosed abdominal pain [4]. Initial US evaluation of the gut should be performed in all four quadrants using curvilinear probe $(3.5-5 \mathrm{MHz})$. The goal is to

\section{A. Laghi}

Department of Radiological, Oncological and Pathological Sciences, Sapienza- University of Rome, ICOT Hospital, Latina, Italy

A. K. Hara $(\bowtie)$

Department of Radiology, Mayo Clinic Arizona,

Phoenix, AZ, USA

e-mail: Hara.amy@mayo.edu identify thickened bowel and/or ancillary findings of inflammation (fluid collections, lymphadenopathy). Higherfrequency probe $(7-12 \mathrm{MHz})$ provides better resolution of the bowel wall layers and surrounding tissues and can be subsequently used to evaluate regions of suspected bowel disease. Color Doppler US provides additional information regarding mural vascularity [5].

CT and MR evaluations of the small bowel require lumen distention, achievable by fluid administration after nasojejunal intubation (CT/MR enteroclysis, CTE) or per os (CT/MR enterography, MRE). Enterography should be preferred, in the setting of inflammatory bowel disorders, due to less invasiveness and similar diagnostic accuracy compared to enteroclysis. Usually for an average-size adult, the dose is around 1500-2000 mL of enteral contrast agent, divided into three aliquots administered starting around $60 \mathrm{~min}$ before CT scanning to reduce patient's discomfort $[1,6]$. Spasmolytic agents should be administered intravenously immediately before the examination, in order to minimize motion artefacts [1]. Intravenous injection of contrast media is mandatory to depict mural enhancement pattern [1].

Advantages of MR imaging over computed tomography (CT) include high contrast resolution, lack of radiation exposure, and use of intravenous contrast media with better safety profiles. MRE also allows dynamic assessment of small bowel peristalsis and distensibility of lumen narrowing, providing functional information [1].

Limitations of MR imaging include costs and variability in examination quality (related to patient cooperation and breath-holding ability), and its role in the emergency setting is still limited [6].

\subsection{Normal Anatomy}

The small bowel has an average length of $6 \mathrm{~m}$ (ranging between 3 and $10 \mathrm{~m}$ ) and includes the duodenum, jejunum (proximal), and ileum (distal). It is attached at the posterior 
abdominal wall thought the mesentery formed by the double fold of peritoneum. Its root extends from the left of L 2 to the right sacroiliac joint and it is $15 \mathrm{~cm}$ long.

The duodenum is the first and shortest segment of the small intestine $(20-30 \mathrm{~cm})$ and continues into the jejunum at the duodenojejunal flexure, fixed to the retroperitoneum by ligament of Treitz. The jejunum occupies the left upper abdomen and constitutes about one third of the small bowel. One of the typical morphological finding in jejunum are the circular mucosal folds, known as valvulae conniventes or plicae semilunaris, more prominent than in the ileum and easily depicted during an MRE or CTE. The ileum occupies the central and right lower part of the abdomen, and the ileocecal valve separates the small from large intestine.

Two main normal anatomical aspects should be considered during the evaluation of small bowel: wall thickness and caliber. The normal caliber of jejunal and ileal lumen, without the administration of spasmolytic agents, must be $<30 \mathrm{~mm}$, and the wall thickness $<3 \mathrm{~mm}$ [1].

\subsection{Pathology}

\subsubsection{Emergency}

US is often the first imaging method for undiagnosed abdominal pain [4]. However, CT is considered the gold standard, offering a more comprehensive evaluation of emergency conditions. Small bowel obstruction (SBO), bowel hemorrhage, and ischemia are easily detected with $\mathrm{CT}$.

SBO continues to be a substantial cause of morbidity and mortality. Adhesions, hernias, and malignancies account for more than $80 \%$ of all SBOs. The task of the radiologist is to determine its site, cause, and the presence or absence of complications such as ischemia or perforation. The hallmark of SBO is dilated small bowel $(>3 \mathrm{~cm})$ proximal to the site of obstruction with decompressed distal bowel. Air-fluid levels and string of beads sign (fluid-filled loops of bowel with a small amount of remaining gas trapped in folds between valvulae conniventes) may be identified. In high-grade obstruction, stasis and mixing of small bowel contents with gas creates the so-called "small bowel feces" sign, an appearance analogous to feces in colon [7] (Fig. 12.1).

The three main causes of mesenteric ischemia are arterial occlusion, venous occlusion, and poor cardiac output or hypovolemia. CT findings associated with ischemic bowel include bowel with thickening, mesenteric edema and fluid adjacent to bowel loops, abnormal decreased bowel wall enhancement, and pneumatosis with or without associated gas in mesenteric or portal veins.

Bleeding in the GI tract has many possible causes, including ulcers, vascular malformations, and tumors. Active small bowel bleeding at multiphase CTE is observed as a gradual accumulation of contrast material within the bowel lumen [8].

\subsubsection{Inflammatory}

\subsubsection{Crohn's Disease (CD)}

One of the most common inflammatory diseases of the small bowel is Crohn's disease (CD) which can involve the entire gastrointestinal tract from the mouth to the anus, most commonly affecting the ileocecal region (approximately $50 \%$ of cases), followed by the ileum (30\%) and colon (20\%) [9]. Perianal disease is present in $20 \%$ of CD patients at initial presentation. CD exhibits skip lesions with transmural inflammation of the bowel wall, deep ulcerations at endoscopy [10], granulomas, and focal crypt irregularity on histology [9].

Cross-sectional imaging of the small bowel with CTE or MRE is performed to identity the presence, length, and severity of inflammation as well as to identify complications such as fistulas, abscesses, and obstruction [11]. CTE and MRE have similar performance for diagnosing CD [12]. CT is more widely available and less time consuming than MR, but MR spares radiation exposure which can be considerable over time [11].

CD phenotypes include quiescent, active inflammatory, stricturing, and penetrating. Due to the chronic and relapsing nature of $\mathrm{CD}$, these phenotypes often coexist (Fig. 12.2a). A primary responsibility of the radiologist is to communicate if there are signs of active inflammation such as mural enhancement, perienteric inflammation, bowel wall thickening/ edema, dilated vasa recta, ulcerations, and restricted diffusion [13]. As these findings are nonspecific and can be seen with a wide variety of other disease processes, the pattern of bowel wall involvement can be helpful. Asymmetric bowel wall involvement that prefers the mesenteric side of the bowel wall predominantly is a pattern that is specific for $\mathrm{CD}$ and not in other disease processes.

Chronic changes of CD include bowel wall fibrosis, strictures, obstruction, and chronic mesenteric venous occlusion. Thus, in addition to identifying signs of active inflammation that could lead to medical therapy, the radiologist must report findings that could result in surgical intervention such as strictures with upstream bowel dilations $>3 \mathrm{~cm}$, fistulas, frank perforation, and abscesses [13].

\subsubsection{NSAID Enteropathy}

Nonsteroidal anti-inflammatory drugs (NSAID) [14] can induce small bowel injury, primarily through direct mucosal injury, resulting in ulcers and eventually characteristic circumferential strictures/diaphragms. Clinically, patients can present with obscure GI bleeding with iron deficiency 

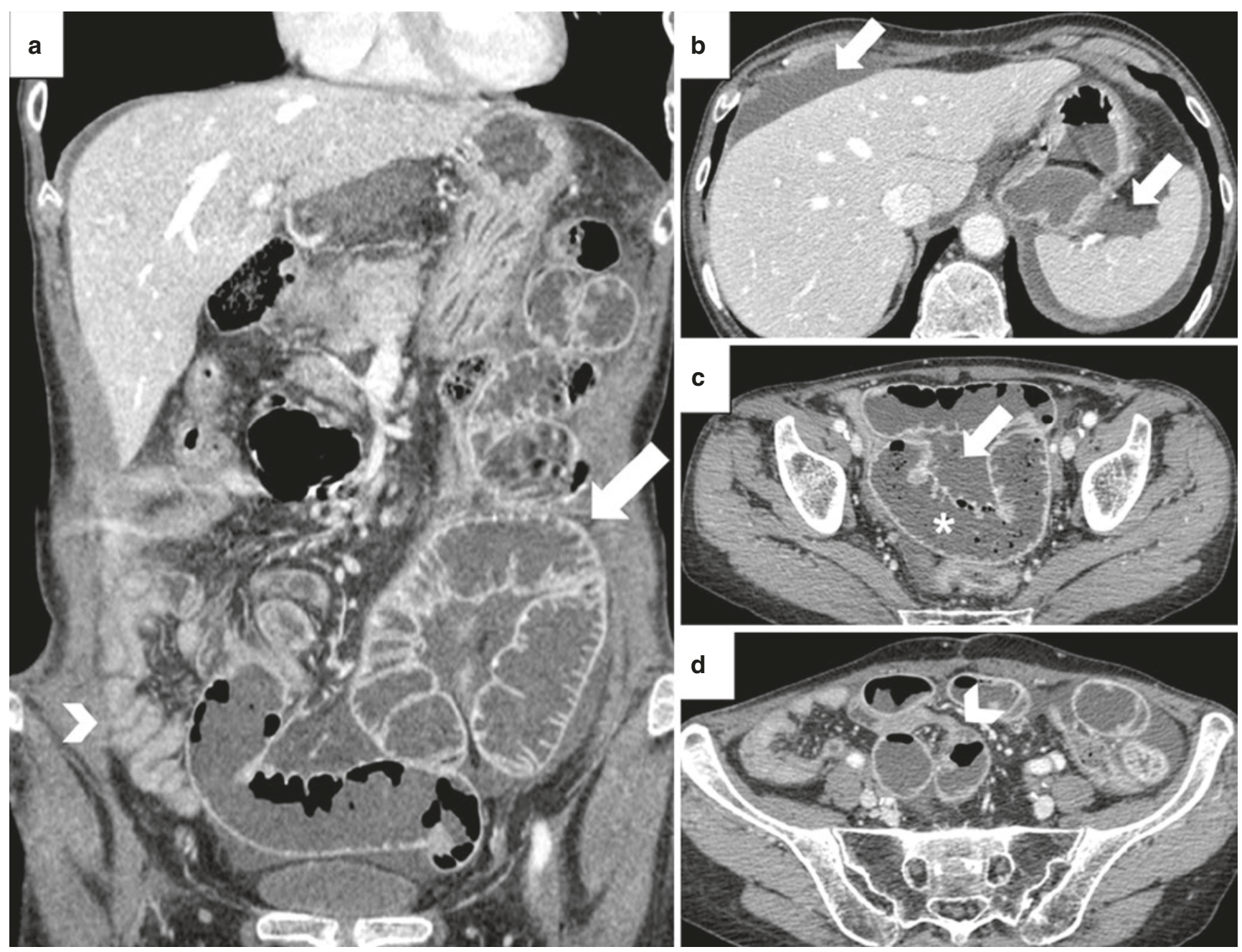

Fig. 12.1 A 55-year-old man with SBO caused by adhesive bands secondary to a prior laparotomy. Contrast enhanced coronal CT reformation shows dilated fluid-filled small bowel (arrow in a) with decompressed distal small bowel (arrowhead) consistent with obstruction. Peritoneal fluid is identified in axial images (arrow in $\mathbf{b}$ and $\mathbf{c}$ ) both in upper and lower abdomen. Obstruction point (arrowhead in d) is identified at the point of transition, immediately below a dilated bowel segment filled with gas bubbles mixed with particulate matter (asterisk in c) (feces sign) anemia, abdominal pain, and bowel obstruction. The gold standard for detecting NSAID enteropathy is capsule endoscopy and balloon enteroscopy. Treatment includes endoscopic dilatation, stricturoplasty, or resection.

These strictures can be challenging to detect at CTE, MRE, and barium exams as they are typically short segment and weblike (Fig. 12.2b) rather than longitudinal strictures seen with CD. Unlike CD, the bowel wall involvement is typically circumferential and does not prefer the terminal ileum. These strictures are commonly multifocal and, depending on the presence of active inflammation, may or may not present with bowel thickening and increased mural enhancement. Without increased mural enhancement and upstream bowel dilatation, a small bowel diaphragm can be difficult to differentiate from a peristaltic contraction or underdistention. In these cases, multiphasic imaging and optimized bowel distention is useful.

Diagnoses that can simulate NSAID enteropathy at imaging are strictures due to radiation, $\mathrm{CD}$, potassium chloride tablets, and eosinophilic gastroenteritis. The diagnosis can be narrowed by the clinical history, bowel location, and other associated radiologic features. For example, fistulas or fatty deposition would favor $\mathrm{CD}$ or involvement of pelvic small bowel loops only favor radiation injury in a patient with that clinical history.

\subsubsection{Celiac Disease}

Celiac disease [15] is an inflammatory small bowel disease triggered by gluten ingestion and typically resulting in diarrhea predominant symptoms but can be asymptomatic and detected by screening for antibodies against tissue transglutaminase. 

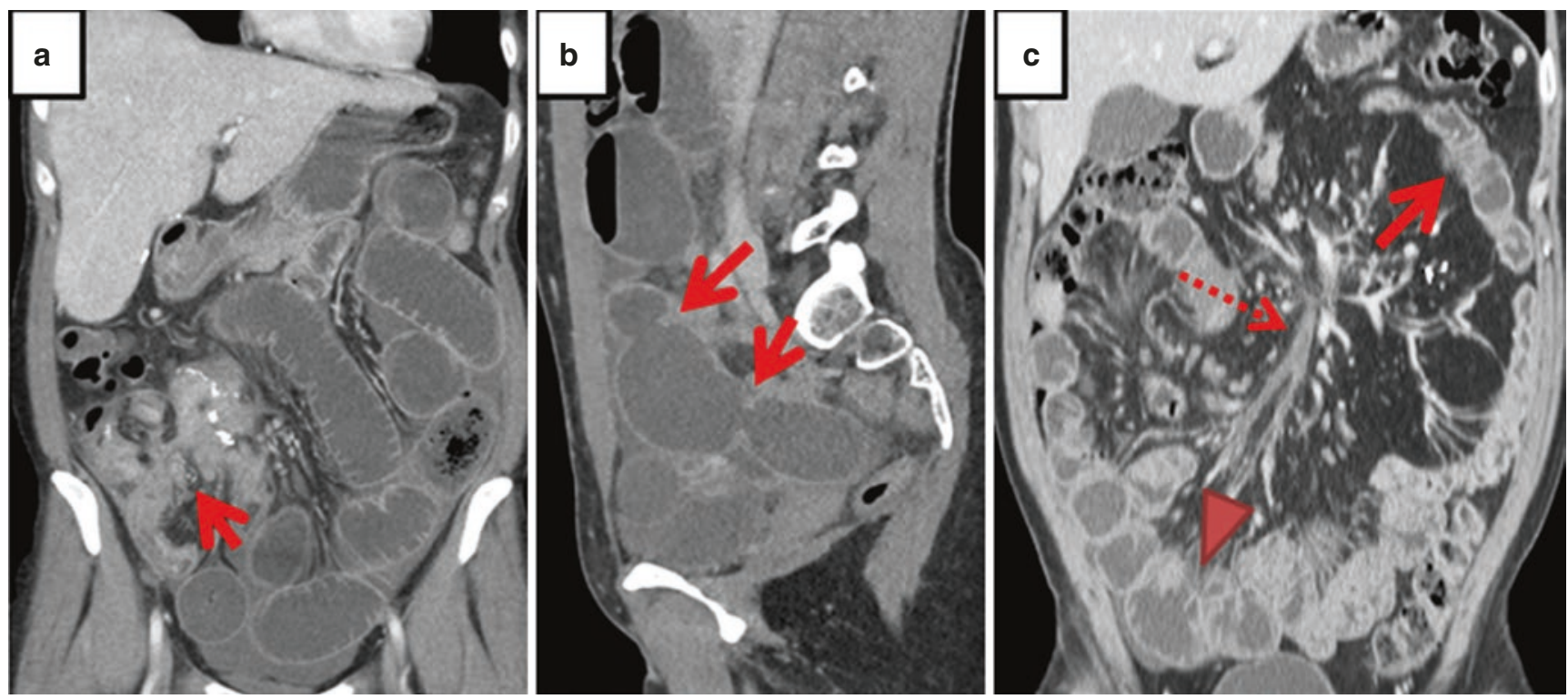

Fig. 12.2 Inflammatory bowel diseases. (a) Crohn's disease showing complex enteroenteric fistula involving the terminal ileum (arrow), active inflammation of involved bowel loops, and strictures with small bowel dilatation. (b) NSAID enteropathy with multiple weblike stric- tures (arrows) and small bowel dilatation. (c) Celiac disease with decreased jejunal folds (arrow), increased ileal folds (arrowhead), and superior mesenteric vein thrombosis (dotted arrow)
The diagnosis is confirmed by biopsy which shows intraepithelial lymphocytes, crypt hyperplasia, and villous atrophy. A subset of patients may have persistent symptoms even with gluten avoidance leading to a diagnosis of refractory celiac disease (RCD). Type $2 \mathrm{RCD}$ is associated with enteropathy associated T-cell lymphoma (EATL) leading to higher mortality.

Imaging features of celiac disease at cross-sectional imaging and barium exams include reversal of the jejunalileal fold pattern (decreased jejunal folds and increased ileal folds) (Fig. 12.2c), intussusception, dilated small bowel, and bowel lymphoma. Additional associated findings at CT/MR are mesenteric/retroperitoneal adenopathy and hyposplenism. Findings of active inflammation as seen in CD can also be present with celiac disease including mural enhancement, bowel wall thickening, and dilated vasa recta.

\subsubsection{Small Bowel Tumors}

Small bowel tumors account for 3-6\% of all GI neoplasms and $1-3 \%$ of all GI malignancies [16]. The most common subtypes are adenocarcinoma (30-45\%), neuroendocrine tumors (20-40\%), lymphoma (10-20\%), and sarcomas (1015\%) [17] (Fig. 12.3). Small bowel tumor incidence has been rising for unclear reasons, although the mortality rate is stable and 5-year survival has been improving [18].

Adenocarcinoma (ACA) is the most common primary malignancy of the small bowel, follows the adenoma-carcinoma sequence and typically arises in the proximal small bowel. Risk factors for ACA include conditions associated with chronic inflammation such as $\mathrm{CD}$, celiac disease, ileostomy or duodenal/jejunal bypass surgery, as well as syndromes such as Peutz-Jeghers and familial polyposis. At imaging, ACA classically has an apple-core appearance associated with bowel obstruction and adenopathy. If it is an intraluminal polypoid mass, it can predispose to intussusception. It typically enhances more than lymphoma but less avidly compared to carcinoid and GI stromal tumors [19].

Carcinoid neuroendocrine tumors (NET) are the second most common primary small bowel malignancy. Unlike ACA, NET most commonly arise in the ileum, are multifocal in $15-35 \%$ of cases and hypervascular. These tumors commonly metastasize to mesenteric lymph nodes resulting in the classic calcified mesenteric mass with associated desmoplastic response, tethering and obstructing adjacent bowel loops, and causing vascular engorgement. Nuclear studies with 68Gallium Dotatate or Indium -111 octreotide, somatostatin analogues, can be used to detect the primary tumor as well as metastatic disease [20].

Lymphoma is the third most common small bowel malignancy and can be the primary site or a site of secondary involvement. The distal ileum is the most common location because it has the most lymphoid tissue. Most cases are non-Hodgkin B-cell lymphoma although T-cell lymphoma is more common in celiac disease and occurs in the jejunum. At imaging, lymphoma can have a wide variety of presentations from mucosal nodules to circumferential bowel wall thickening with aneurysmal dilatation to a large exocentric mass [19].

GI stromal tumors arise from the interstitial cells of Cajal, intestinal pacemaker cells that line the GI tract [21]. They are initiated by mutations in KIT, a tyrosine kinase growth factor receptor, which allows the cells to grow and divide 

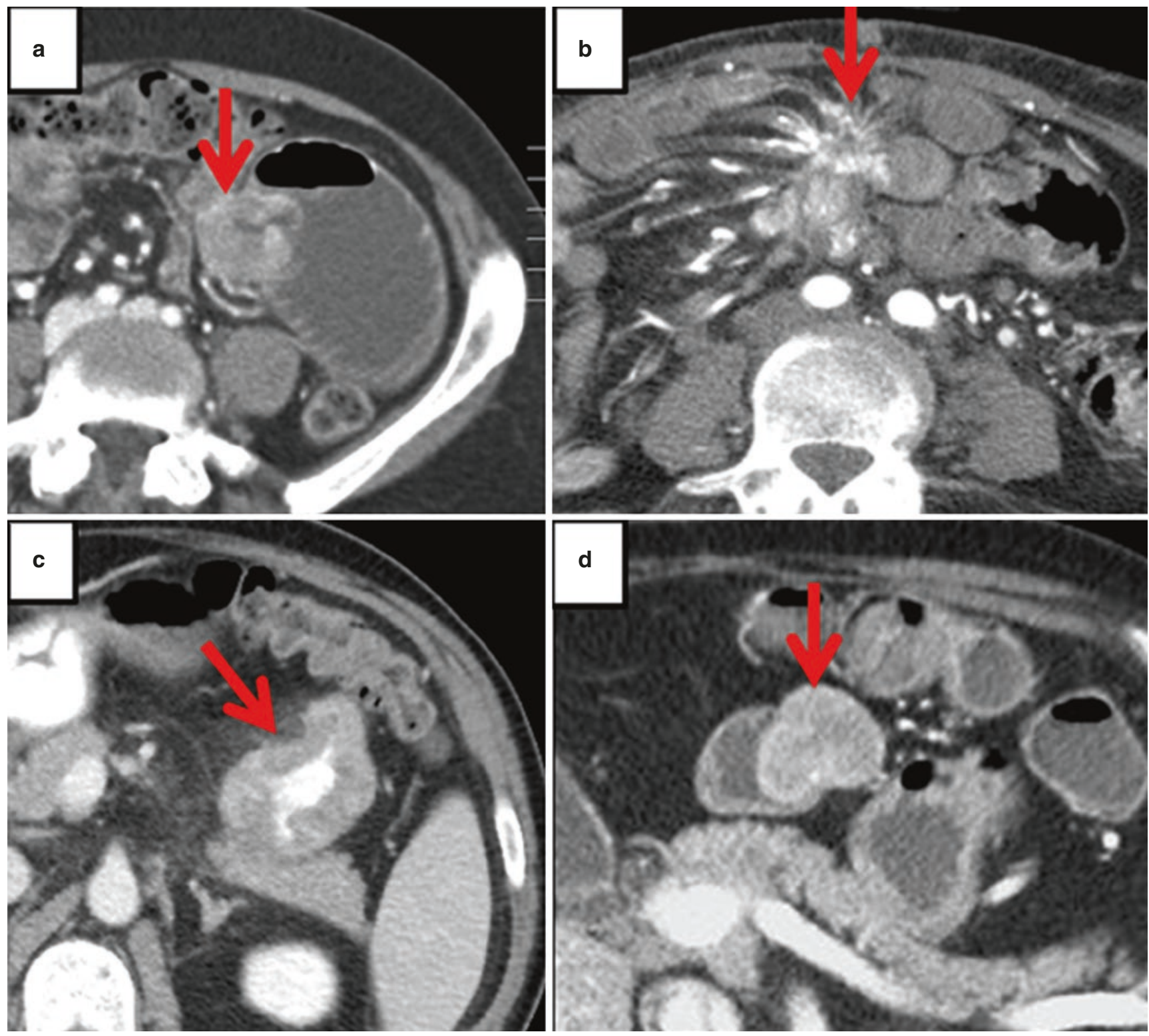

Fig. 12.3 Small bowel tumors at CT. (a) Adenocarcinoma (arrow) with mild enhancement and associated small bowel obstruction. (b) Carcinoid (arrow) with mesenteric mass, associated bowel obstruction, desmoplastic response, and vascular engorgement. (c) Jejunal lym-

abnormally. Thus, they can be treated by tyrosine kinase inhibitors such as imatinib mesylate. These tumors can have a wide variety of imaging appearances, presenting as a homogeneously hypervascular enhancing small bowel mass to a large hypoenhancing exophytic cavitary mass. They are typically large at presentation as they don't commonly cause bowel obstruction, unlike ACA and NET. Surgical resection is typically the treatment of choice. Target therapies with tyrosine kinase inhibitors are used if not surgically resectable or to prevent recurrence/metastases. PET/CT can be used to evaluate treatment response as size criteria alone can underestimate response. phoma (arrow) with bowel wall thickening and preservation of the lumen (aneurysmal dilatation). (d) GI stromal tumor (arrow) with typical lobulated, homogenous hypervascular appearance without bowel obstruction

\subsection{Concluding Remarks}

Diagnostic imaging and in particular cross-sectional modalities (US, CT, and MR) have a critical and complementary role in diagnosis and management of small bowel diseases. Radiologists should be aware of advantages and disadvantages of each imaging test in order to choose the best option, considering the specific small bowel disease and the patient's characteristics (age, gender, clinical status). A deep knowledge of the different entities involving the small bowel is also critical in order to reach a correct diagnosis and to provide the necessary information for patient management. 


\section{Take-Home Messages}

- A small bowel loop is considered normal when caliber is $<30 \mathrm{~mm}$ and wall thickness $<3 \mathrm{~mm}$.

- US is a powerful diagnostic test for bowel assessment in patients with known or suspected gutrelated disease, especially in combination with the administration of oral and/or intravenous contrast agents.

- $\mathrm{CT}$ is the imaging modality of choice in the emergency setting (i.e., small bowel occlusion, ischemia, and, in some circumstances, bleeding).

- MR is the preferred imaging modality in inflammatory bowel diseases (IBD) because of multiparametric approach, evaluation of bowel motility, and lack of radiation exposure.

- In IBD, cross-sectional imaging is performed to identity the presence, length, and severity of inflammation as well as to assess complications such as fistulas, abscesses, and obstruction.

- Imaging features of celiac disease at cross-sectional imaging and barium exams include reversal of the jejunal-ileal fold pattern (decreased jejunal folds and increased ileal folds), intussusception, dilated small bowel, and bowel lymphoma.

- Small bowel tumors account for 3-6\% of all GI neoplasms and 1-3\% of all GI malignancies; in order of frequency, they are adenocarcinoma (30-45\%), neuroendocrine tumors $(20-40 \%)$, lymphoma (10$20 \%$ ), and sarcomas (10-15\%).
3. Serafin Z, Białecki M, Białecka A, Sconfienza LM, Kłopocka M. Contrast-enhanced ultrasound for detection of Crohn's disease activity: systematic review and meta-analysis. J Crohns Colitis. 2016;10:354-62.

4. Laméris W, van Randen A, van Es HW, et al. Imaging strategies for detection of urgent conditions in patients with acute abdominal pain: diagnostic accuracy study. BMJ. 2009;338:b2431.

5. Muradali D, Goldberg DR. US of gastrointestinal tract disease. Radiographics. 2015;35:50-68.

6. Murphy KP, McLaughlin PD, O'Connor OJ, Maher MM. Imaging the small bowel. Curr Opin Gastroenterol. 2014;30:134-40.

7. Paulson EK, Thompson WM. Review of small-bowel obstruction: the diagnosis and when to worry. Radiology. 2015;275:332-42.

8. Nakashima K, Ishimaru H, Fujimoto T, et al. Diagnostic performance of CT findings for bowel ischemia and necrosis in closedloop small-bowel obstruction. Abdom Imaging. 2015;40:1097-103.

9. Hart A, Ng SC. Crohn's disease. Medicine. 2011;39:229-36.

10. Shergill AK, Lightdale JR, Bruining DH, et al. The role of endoscopy in inflammatory bowel disease. Gastrointest Endosc. 2015;81:1101-21.e1-1101-21.e13.

11. Panes J, Bouhnik Y, Reinisch W, et al. Imaging techniques for assessment of inflammatory bowel disease: joint ECCO and ESGAR evidence-based consensus guidelines. J Crohns Colitis. 2013;7:556-85.

12. Horsthuis K, Stokkers PC, Stoker J. Detection of inflammatory bowel disease: diagnostic performance of cross-sectional imaging modalities. Abdom Imaging. 2008;33:407-16.

13. Deepak P, Park SH, Ehman EC, et al. Crohn's disease diagnosis, treatment approach, and management paradigm: what the radiologist needs to know. Abdom Radiol. 2017;42:1068-86.

14. Frye JM, Hansel SL, Dolan SG, et al. NSAID enteropathy: appearance at CT and MR enterography in the age of multi-modality imaging and treatment. Abdom Imaging. 2015;40:1011-25.

15. Tennyson CA, Semrad CE. Small bowel imaging in celiac disease. Gastrointest Endosc Clin N Am. 2012;22:735-46.

16. Pan SY, Morrison H. Epidemiology of cancer of the small intestine. World J Gastroint Onc. 2011;3:33-42.

17. Sokhandon F, Al-Katib S, Bahoura L, et al. Multidetector CT enterography of focal small bowel lesions: a radiologicalpathological correlation. Abdom Radiol. 2017;42:1319-41.

18. National Cancer Institute. SEER stat fact sheets: small intestine cancer. Available at: http://seer.cancer.gov/statfacts/html/smint. html.

19. Buckley JA, Fishman EK. CT evaluation of small bowel neoplasms: spectrum of disease. Radiographics. 1998;18:379-92.

20. Yu R, Wachsman A. Imaging of neuroendocrine tumors: indications, interpretations, limits, and pitfalls. Endocrinol Metab Clin N Am. 2017;46:795-814.

21. Scola D, Bahoura L, Copelan A, et al. Getting the GIST: a pictorial review of the various patterns of presentation of gastrointestinal stromal tumors on imaging. Abdom Radiol. 2017;42:1350-64. enteroclysis, in characterizing lesions in patients with Crohn's disease. Clin Gastroenterol Hepatol. 2013;11:950-5.

1. Taylor SA, Avni F, Cronin CG, et al. The first joint ESGAR/ ESPR consensus statement on the technical performance of cross-sectiona small bowel and colonic imaging. Eur Radiol. 2017;27:2570-82.

2. Calabrese E, Zorzi F, Onali S, et al. Accuracy of small-intestine contrast ultrasonography, compared with computed tomography

Open Access This chapter is licensed under the terms of the Creative Commons Attribution 4.0 International License (http://creativecommons. org/licenses/by/4.0/), which permits use, sharing, adaptation, distribution and reproduction in any medium or format, as long as you give appropriate credit to the original author(s) and the source, provide a link to the Creative Commons license and indicate if changes were made.

The images or other third party material in this book are included in the book's Creative Commons license, unless indicated otherwise in a credit line to the material. If material is not included in the book's Creative Commons license and your intended use is not permitted by statutory regulation or exceeds the permitted use, you will need to obtain permission directly from the copyright holder. 